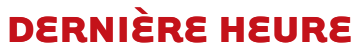

\section{Un allèle IGFI pour les petits chiens}

Simone Gilgenkrantz
Médecine/Sciences, 9, rue Basse, 54330 Clérey-sur-Brénon, France. sgilgenkrantz@medecinesciences.org

même génome, mais avec une sélection d'allèles différents ${ }^{2}$. Pour analyser cette diversité génétique, l'identification de quelque 500000 sites génétiques polymorphes (SNP, single nucleotide polymorphism) a été effectuée à partir de diverses races de chiens et constitue une ressource incomparable. Car en privilégiant l'analyse des polymorphismes génétiques, Canis familiaris offre ainsi un modèle animal extrêmement précieux pour la compréhension du génome normal et pathologique des mammifères.

\section{La taille dans les races canines}

Un groupe de chercheurs anglo-saxons vient d'en apporter la preuve en démontrant le rôle majeur d'un des allèles du gène IGFI (insulin like growth factor) expliquant les variations de taille des races canines [2]. Des travaux préliminaires avaient été présentés en octobre 2006 au meeting annuel de l'American Society of Human Genetics, mais la publication récente dans Science détaille une méthode d'analyse exemplaire. Elle pourra servir de modèle pour mesurer les variations d'autres caractères et permettre ainsi au chien de se rendre encore, d'une autre manière, utile à l'homme.

Dans un premier temps, l'étude a porté sur le Chien d'eau Portugais (PWD), une race existant depuis des siècles sur les côtes du Portugal: le PWD se plaît à accompagner les pêcheurs en mer (Figure 1). Bon nageur et bon plongeur,

\footnotetext{
${ }^{2}$ Voir la couverture du Magazine Science du 6 avril 2007 un Chihuahua à côté d'un Grand Danois (qui fait 50 fois sa masse).
} 
il les aide à ramener leurs filets. Mais surtout, la définition de la race, en matière de taille, est tolérante et a été déterminante pour le choix de l'animal. En effet, des variations de taille assez importantes sont observées et acceptées, en particulier par l'American Kennel Club qui sert de référence aux États-Unis en la matière. Les radiographies squelettiques de 92 PWD ont donc été mesurées, et deux QTL (quantitative trait loci) sur le chromosome 15 canin se sont révélé être associés à la taille. Puis, sur 463 PWD, l'analyse a confirmé l'étroite corrélation entre la taille des animaux et l'un de ces $\mathrm{PTL}$, dans lequel se trouve le gène IGFI. Ce gène, on le sait, joue un grand rôle dans la taille des souris et des hommes (bien que les disparités soient infiniment moindres que chez les chiens). Après avoir mis en évidence plusieurs centaines de SNP, le classement de 116 d'entre eux montra un seul pic correspondant à la région du gène IGFI [3].

En déterminant l'haplotype de 20 SNP autour d'IGFl, il apparut que, sur 926 PWD, 889 d'entre eux (96\%) ne possédaient que deux haplotypes (appelés $B$ et $I$ ) et que les chiens BB étaient plus petits et avaient des concentrations en protéine IGFl (ou somatomédine C) plus basses que celles des chiens homozygotes II. Toutefois, le déséquilibre de liaison autour d'IGFI chez les PWD était trop important pour permettre

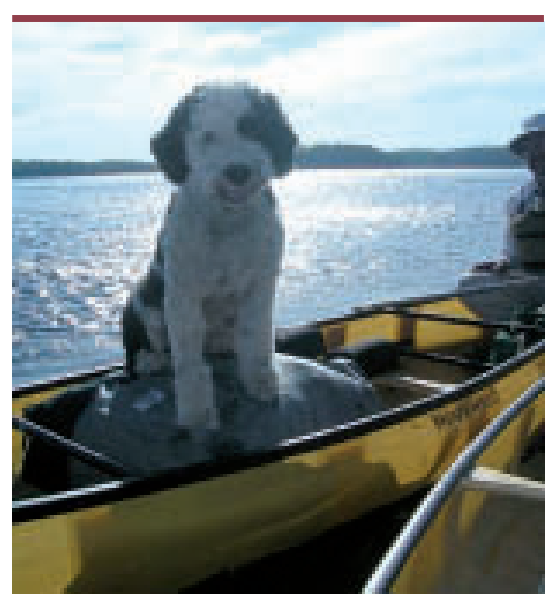

Figure 1. Chien d'eau Portugais. une cartographie plus fine, sans doute parce que la création de la race est trop récente [3]. Mais en admettant qu'une mutation intervienne dans la taille des chiens, l'étude comparative de chiens d'autres races, de taille différente et ayant par ailleurs des histoires généalogiques indépendantes, devait permettre de vérifier le rôle d'IGFI, d'autant plus que la taille a toujours été un facteur de sélection important pour la création des races canines.

\section{Du côté des petits chiens}

On pouvait donc s'attendre à trouver, dans le QTL sélectionné, des différences entre les races de petits chiens et celles des très grands. Les 116 SNP ont donc été analysés chez 526 chiens de race, allant des Chihuahua aux Saint Bernard et aux grands Danois. Quatre-vingt trois autres SNP, situés sur d'autres chromosomes et n'ayant aucune relation avec la taille des animaux ont été utilisés comme témoins afin d'établir une distribution empirique pour les analyses statistiques.

Les résultats montrent qu'un même haplotype est retrouvé dans la quasi totalité des races de petite taille, identique à l'haplotype $B$ des PWD. En revanche, d'autres haplotypes, surtout I et $F$, sont retrouvés dans les grandes races. Vingt marqueurs situés dans la région du gène IGFI ont ensuite été retenus et séquencés. Les haplotypes, allant de $A$ à $L$, sont diversement répartis. L'haplotype $G$ devait correspondre aux populations originelles car il est retrouvé chez le loup gris (C. lupus), le chacal commun (C. aureus) et le coyote (C. latrans). En revanche, l'haplotype $A$, exceptionnel chez les grands chiens, est extrêmement fréquent chez les chiens de petite race. Parmi les marqueurs de la région d'IGFl, le SNP 5 a été retenu comme le plus proche de la mutation causale car: (1) il comporte les haplotypes A, B et $C$ associés aux petites races; (2) il n'y a pas de recombinaison entre ce SNP 5 et le SNP 4 ; (3) il est retrouvé chez le loup gris, le chacal commun et le coyote, montrant qu'il provient bien des races originelles de canidés.

Les analyses génétiques ont alors porté sur plus de 3000 chiens appartenant à 143 races différentes. Les résultats sont probants. L'association entre l'allèle SNP5 A et la taille est attestée par plusieurs études statistiques à deux exceptions près: les Rottweiler (d'origine allemande) et les Mastiff (d'origine anglaise, très ancienne) pour lesquels des études ultérieures devront être entreprises (Figure 2).

\section{Le gène IGFI}

Le gène IGFI est un déterminant génétique important de la taille des mammifères. Les souris déficientes en IGFI ont un poids de naissance correspondant à $60 \%$ du poids normal. Chez l'homme, des délétions d'IGFI (qui se situe en 12q22q24.1) ont été rapportées avec une taille à la naissance de moins 3,9 déviations standard [4]. Chez les caniches, la concentration en protéine IGFl est en relation avec la taille des différentes races: caniches royal, standard, nain, ou toy [5]. Les PWD ayant I'haplotype B ont une concentration en somatomédine C (ou IGFl) moins élevée. Le séquençage du gène IGFI (les exons, certains introns et le promoteur) a été effectué dans différentes races de chiens, avec des différences en fonction de la taille, mais le variant causal n'a pu être déterminé.

\section{Conclusions}

L'ensemble des résultats fait donc penser que le gène IGFI a substantiellement contribué à la variation de taille des chiens domestiques. Puisque toutes les petites races étudiées, dont les histoires sont très éloignées les unes des autres, portent le même haplotype, il est probable que la mutation est survenue très tôt dans l'histoire de l'espèce canine.

Les ossements canins les plus anciens datent d'environ 12 à 15000 ans [6] et montrent que la diversité des tailles existait déjà au moment de la domestication. Ceux qui ont été retrouvés en Russie correspondent à des squelettes 


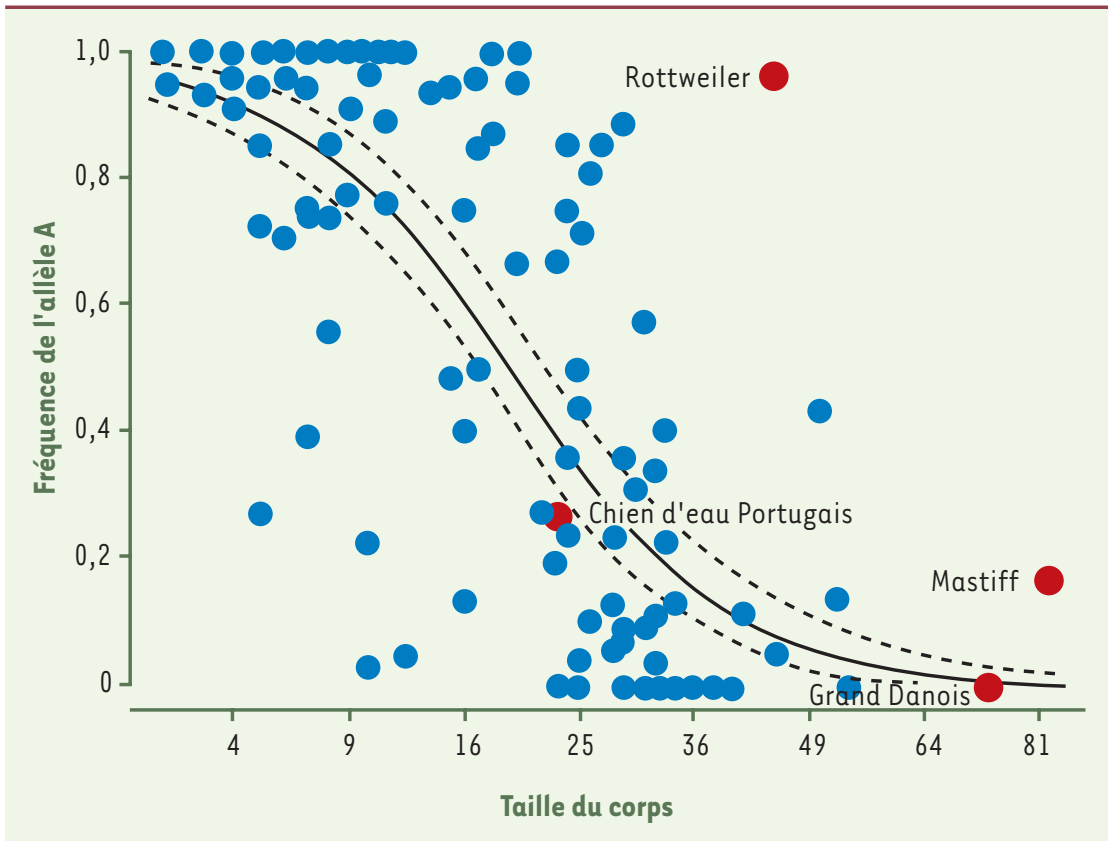

Figure 2. Courbes de régression binomiale de la fréquence de l'allèle SNP5 A sur la racine carrée de la masse moyenne. Les pointillés indiquent l'intervalle de confiance ( $95 \%$ ). Entre 5 et 143 chiens (moyenne $=22$ ) ont été génotypés pour chacune des 143 races (d'après [3]).

des chiens proches des grands Danois (60 à $70 \mathrm{~kg}$ ). Ceux qui ont été découverts au Moyen-Orient et en Europe sont voisins des Terriers ( 7 à $9 \mathrm{~kg}$ ); ils sont beaucoup plus nombreux et se trouvent dans des zones géographiques beaucoup plus étendues. Cet avantage aux petits chiens a fait supposer que la modification d'IGFl est survenue très tôt et qu'elle a été maintenue par sélection. Certains auteurs s'interrogent pour savoir si elle fut intentionnelle ou si elle a résulté d'une adaptation à la coexistence avec les populations humaines [7]. La possibilité d'identifier un gène intervenant dans la morphologie en s'appuyant uniquement sur les races de chiens existant aujourd'hui est ainsi démontrée. II faut toutefois souligner que ce type de recherche représente un travail considérable et qu'il faudra bien choisir l'hypothèse de départ pour qu'il puisse encore porter ses fruits. Mais il peut fournir de précieux renseignements sur la morphogenèse des mammifères, l'homme en particulier. On peut se demander - et on n'ose l'imaginer - si une reproduction dirigée, infligée à d'autres espèces de mammifères, nous fournirait aujourd'hui le spectacle d'une telle diversité. N'existe-t-il pas, dans l'espèce canine, une plasticité particulière? Car la «création» de bien des chiens de race à la mode est récente et n'a nécessité que quelques siècles et parfois moins ${ }^{3}$. Si c'est le cas, la réponse se trouve peut-être dans des études voisines de celle qui est rapportée ici. Quant à l'homme, malgré les barrières culturelles et géographiques, sa liberté de procréation le conduit à la panmixie et les isolats sont de plus en plus rares dans les sociétés modernes. N'oublions pas, toutefois, la «dame de Flores ». Les ossements découverts dans l'île de Florès en Indonésie, provenant d'êtres humains vivant il y a environ 18000 ans, attestent de la très petite taille (un mètre environ) d'Homo floresiensi dont l'existence reste encore mystérieuse [8]. $\diamond$

${ }^{3}$ L'épagneul Papillon, par exemple, est, parmi les épagneuls, une des races les plus récentes. Apparue à la fin du XIXe siècle, cette variété serait l'aboutissement de croisements entre Phalène, Chihuahua, Spitz.
IGF1, an allele for the small dogs

\section{RÉFÉRENCES}

1. Galibert F, André C, Hitte C. Le chien, modèle pour la génétique des mammifères. Med Sci (Paris) 2004 ; $20: 761-6$.

2. Sutter NB, Bustamante CD, Chase $K$, et al. A single allele is a major determinant of small size in dogs. Science 2007 ; 316 : 112-5.

3. Sutter NB, Eberle MA, Parker HG, et al. Extensive and breed-specific linkage disequilibrium in Canis familiaris. Genome Res 2004 ; 14 : 2388-96.

4. Woods KA, Camacho-Hubner C, Savage MO, Clark AJL. Intrauterine growth retardation and postnatal growth failure associated with deletion of the insulin-like growth factor I gene. $N$ Engl J Med 1996 ; 335 : 1363-7.

5. Eigenmann JE, Patterson DF, Froesch $\varepsilon R$. Body size parallels insulin-like growth factor I levels but not growth hormone secretory capacity. Acta Endocrinol $1984 ; 106: 448-53$.

6. Olsen SJ. Origins of the domestic dog. Tucson, AZ : University of Arizona Press, 1985.

7. Tchernov $\varepsilon$, Horwitz LK. Body size diminution under domestication: unconscious selection in primeval domesticates J Anthropol Archeol 1991 ; 10 : 54-75.

8. Balter M. Small brains, big fight: Hobbits called new species. Science 2007 ; $315: 583$.

\section{REMERCIEMENTS}

Je remercie Bertrand Jordan pour ses conseils.

\section{PRIX DESCARTES-HUYGENS}

LedocteurHubertVaudry(InsermU413)

- membre du comité de direction de

Médecine/Sciences - co-organisateur

de «La semaine du cerveau»

de Rouen et administrateur de

Science-Action Haute-Normandie, a reçu le Prix Descartes-Huygens 2006 de l'Académie Royale des Arts et Sciences des Pays-Bas.

Ce Prix, créé en 1993 par les gouvernements français et néerlandais, a pour objet de récompenser chaque année deux scientifiques de très haut niveau, de l'une et l'autre nationalité, qui se sont distingués par la qualité de leurs travaux et par leur contribution au renforcement des liens de coopération entre les deux pays. Le prix est attribué en alternance entre les grands secteurs disciplinaires. En 2006, il concernait le domaine des sciences de la vie. 